malformations. For each malformation the incidence is given, followed by simple aids to diagnosis or measurement. A list of syndromes in which the minor malformation is commonly found is given and a note on the overall clinical significance is also provided. This section is the most useful part of the book and provides much information of interest. The lists of syndromes where each specific minor malformation is commonly found are not exhaustive. However, they do cover the more common malformation syndromes. An alphabetical index would have been useful.

In summary, this is a useful book to have on one's shelf to look up the incidence of various minor malformations and to check on methods of measuring or recording these defects. It will not be very useful as an aid to diagnosis of dysmorphic syndromes, but does provide some insight into the clinical significance of minor malformations.

ROBIN M WINTER

\section{Mobile Genetic Elements}

Edited by James A Shapiro. (Pp xvi + 688; figures + tables. $\$ 65$, £43.) London, New York: Academic Press. 1983.

This volume outlines the exciting developments in what is known about the natural history and molecular basis of mobile genetic elements in prokaryotic and eukaryotic organisms. A detailed and comprehensive review of the present state of knowledge and reference list is provided for the various known mobile genetic elements starting, as it should, with the controlling elements in maize, the bacteriophages lambda and $\mathrm{Mu}$, the non-viral mobile elements in bacteria (the insertion sequence (IS) elements and the transposons, Tn3 and Tn10, which confer drug resistance to bacteria), the transposable elements in yeast, the dispersed repetitive DNAs (copia-like, fold-back, and P elements), and hybrid dysgenesis in Drosophila and Agrobacterium tumour induction (crown gall tumour). Four final chapters on retroviruses, flagellar phase variation on Salmonella, yeast mating types, and antigenic variation in Trypanosomes are included because they involve DNA sequences which display behaviour and possess characteristics typical of mobile elements, although they are not always considered in discussions on this topic.

A stated intent of the editor of the text is to enable the non-specialist to begin to understand the biology and genetics of mobile genetic elements. This objective is not achieved in certain cases because although each chapter starts with an introductory section outlining the biology of the particular genetic element being considered, it occasionally requires a greater degree of background knowledge than the non-specialist might be expected to possess in order to be able to understand the experimental details of the system being discussed. Although most chapters include some discussion of similarities in the biology and molecular basis with other mobile genetic elements, the text is not one which can easily be read from cover to cover in the expectation of providing an overview on the whole subject. Each chapter does provide, however, an excellent starting point for a detailed enquiry into what is known about any particular mobile genetic element.

The descriptions of the behaviour, biology, and molecular basis of these mobile genetic elements provokes questions about our understanding of chromosomal structure, genome stability/instability, and gene regulation. Although one is warned several times in the text that our understanding of these elements in eukaryotic systems is still in a relatively primitive state, one cannot help but speculate about their possible role in the reprogramming, restructuring, and relocation of genes and possible aetiology in some syndromes and single gene and chromosomal disorders. Overall, this is a text for the partly initiated rather than the uninitiated.

R F Mueller 